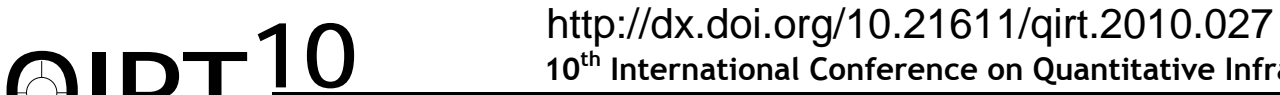 \\ $10^{\text {th }}$ International Conference on Quantitative InfraRed Thermography \\ July 27-30, 2010, Québec (Canada)
}

\section{Detection of foreign bodies in comestible product using sequence of low contrast thermographic images}

\author{
by B. Bukowska-Belniak*, A. Leśniak*, A. Stasz ${ }^{\star \star}$, P. Kiełkowski** and R. Michalski* \\ ${ }^{*}$ Geoinformatics \& Applied Computer Science Dept., AGH University of Science and Technology, Krakow, Poland, \\ bukowska@agh.edu.pl, lesniak@agh.edu.pl \\ **Flying Fish Technology, Krakow, Poland, andrzej.stasz@qnc.pl, piotr@mspnet.eu, r.michalski@mspnet.eu
}

\begin{abstract}
This paper presents the results of sequence of thermographic images processing. The images illustrate cooling process using cold air of some comestible product. The product is contaminated with unwanted objects, which are visible during air blast. Low contrast is characteristic for this sequence of thermographic images, what makes difficult to localize boundaries of the contamination. As a result of the presented analysis methodology of proper detection of those boundaries is showed.
\end{abstract}

\section{Introduction}

In recent years many new applications of thermography has been emerged. This method is especially useful for monitoring natural and artificial processes, for which are observed thermal anomalies. The thermographic methods are especially useful if the remote and non-invasive observation is necessary. The special objects that are subjected inspection are food products. Purity of such products and lack of unwanted contaminants is especially important in case of such products. The reliable methods of inspection are crucial during a production process. Some grocery products are exposed to high temperature because of technological requirements. During its forming or assembling they are heated and then cooled. If thermal conduction values of products and contaminants are different it is possible in some special conditions to detect and localize alien objects inside the product. In such case thermographic inspection of the product gives a potential possibility of online inspection. Because the contaminants cannot sometimes be visible on surface and thermal properties of contaminant and the product can be similar the temperature anomalies are usually small in size and amplitude. The main issue of this paper is to find effective algorithm of thermographic images processing to localize that anomalies in the images and at the same time unwanted objects inside products.

\section{Experiment description}

To perform experiment of remote detection of contaminants in food we have designed the special sample. We have prepared the chocolate bar and filed it with a few alien bodies (stone, piece of glass, piece of plastic). They were located at different depths below the surface and invisible. Then we heat up the bar to reach temperature equal to $26{ }^{\circ} \mathrm{C}$. Next the products were cooled by cold air blast to achieve temperature equal to $16^{\circ} \mathrm{C}$.

As a result we have obtained sequence of twenty thermographic images. They were registered in time interval 5 seconds using VIGOcam v50 thermographic camera, which is produced by Polish company Vigo System S.A. The spatial resolution of images is $288 \times 384$ pixels, the temperature resolution is $0.08{ }^{\circ} \mathrm{C}$ [1].

The sequence of images is presented in figure 1. Two samples of the product on every image were registered - the one with contaminations on the right side of the image and the second without contamination on the left side. The sequence starts at high temperatures and finishes at lower one. During this process, the contaminations start to be visible in infrared band. Maximum temperature registered on images in the sequence is equal to $25.26{ }^{\circ} \mathrm{C}$ and minimum is equal to $18.04{ }^{\circ} \mathrm{C}$. The range of measured temperatures is not high, what characterizes images as low contrastive. Differences in temperatures between comestible product and contamination are maximally equal to $0.7^{\circ} \mathrm{C}$. The alien objects are hardly visible only on few thermographic images. 


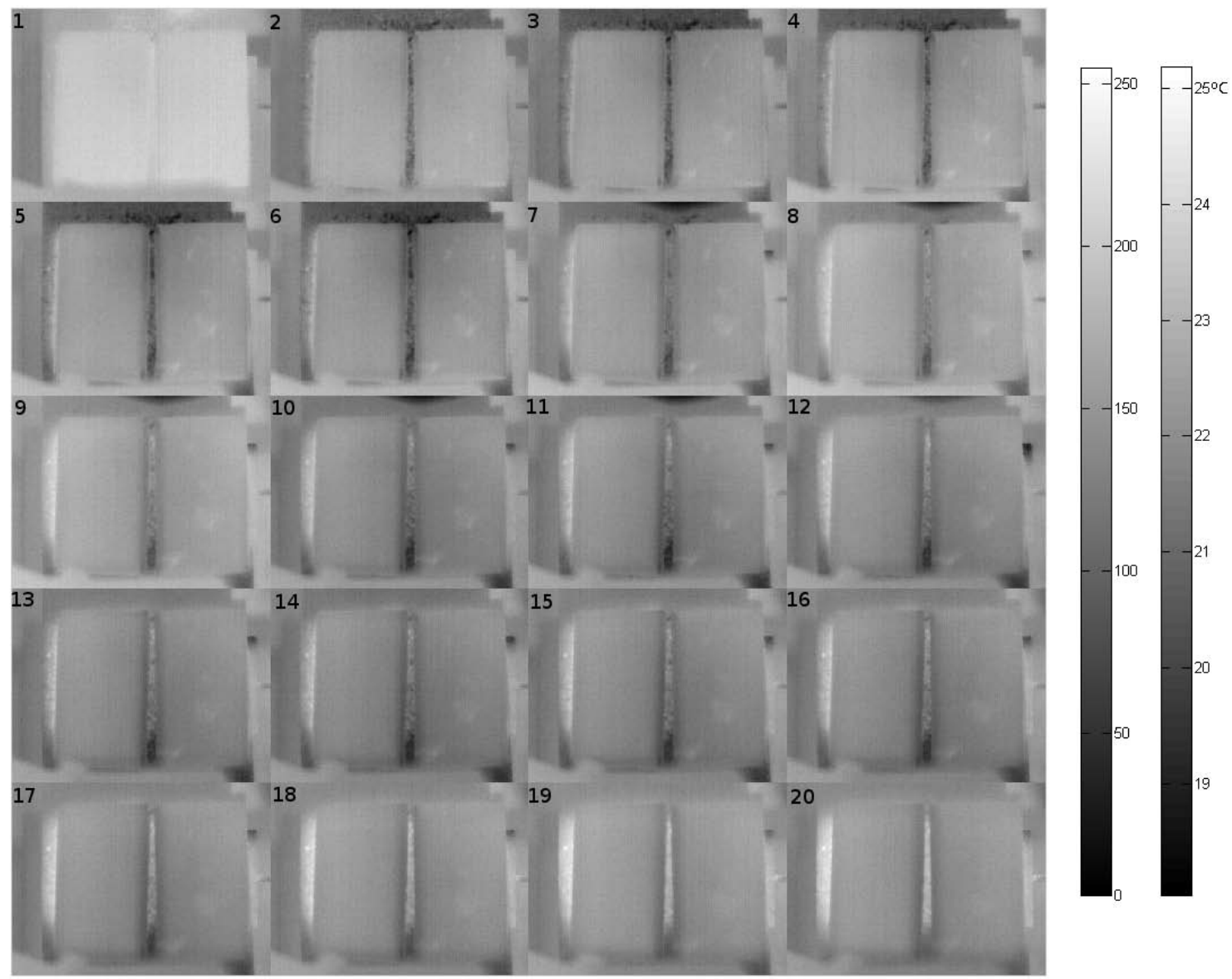

Fig. 1. Sequence of 20 images with visible contaminations during air blast - images 2-17

\section{Image processing}

Images were processed in MATLAB 2007 environment. Scheme of processing was divided into two blocks. In first, object detection was based on images chosen by visual perception, in second, it used those results to point the most contrastive images.

Firstly, the visual view of sequence was made. For next processing we took only the part of the origial images. Selected region has size $220 \times 120$ pixels and presents the right part of the original images from the sequence - the sample with contamination. Next we chose four images from sequence (number 5, 6,7 and 8), which are the most contrastive and in which contaminations are the most visible. For those images we calculated arithmetical mean image to strenght the contamination areas and reduce noise (figure 2a). Then we made thresholding based on mean value on this image (figure $2 \mathrm{~b}$ ) but the results were not satisfactory. It was caused by the background character, which was not constant on the whole image. We used imtophat MATLAB function, which performs morphological top-hat filtering to correct uneven and dark background (figure 2c). In next step we used median filter to reduce noise on the image (figure 2d). Then we made another morphological operation - opening (figure 2e). On the end we made thresholding, closing and segmentation of the image. In all morphological operations we used disk-shaped structuring element. As a result (figure 2f) we obtained binary image, in which white colour corresponds to the contamination and black to the background [2]. 

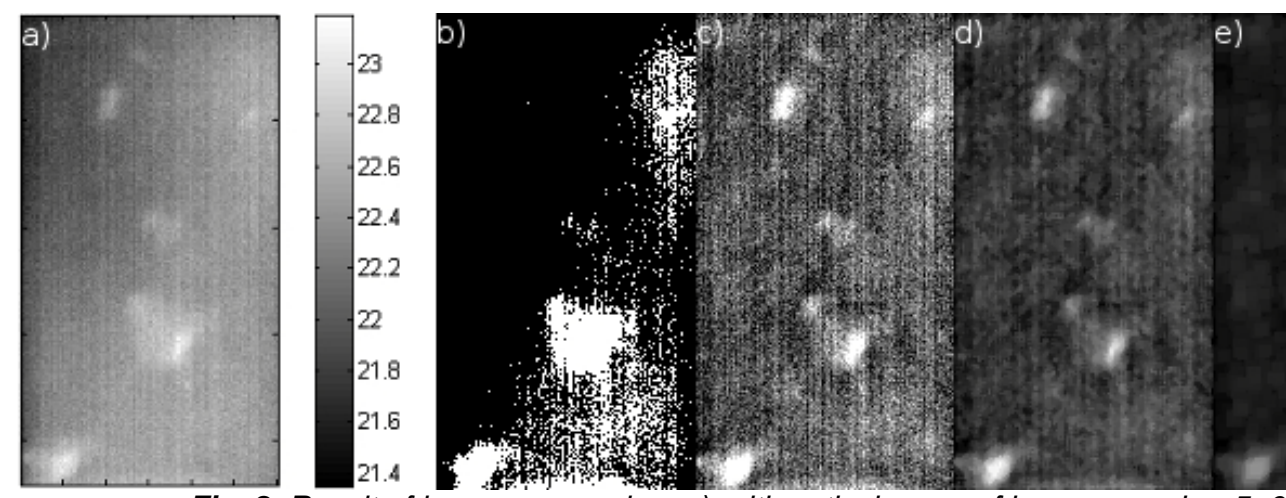

e) f)

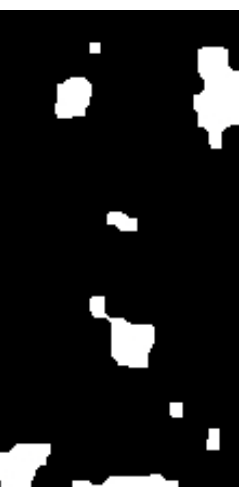

Fig. 2. Result of image processing: a) arithmetical mean of images number $5,6,7$, and 8 ; b) image (a) after thresholding; c) image (a) after morphological top-hat filtering; d) image (c) after median filtering; e) opening of image (d); f) image (e) after thresholding and closing operation

For this result we calculated changes in temperatures of selected regions with and without contamination for all images in the sequence. Figure 3 presents those changes. As we can see the highest differences in temperature between the background and contamination are in images number 3, 4, 5 and 6.

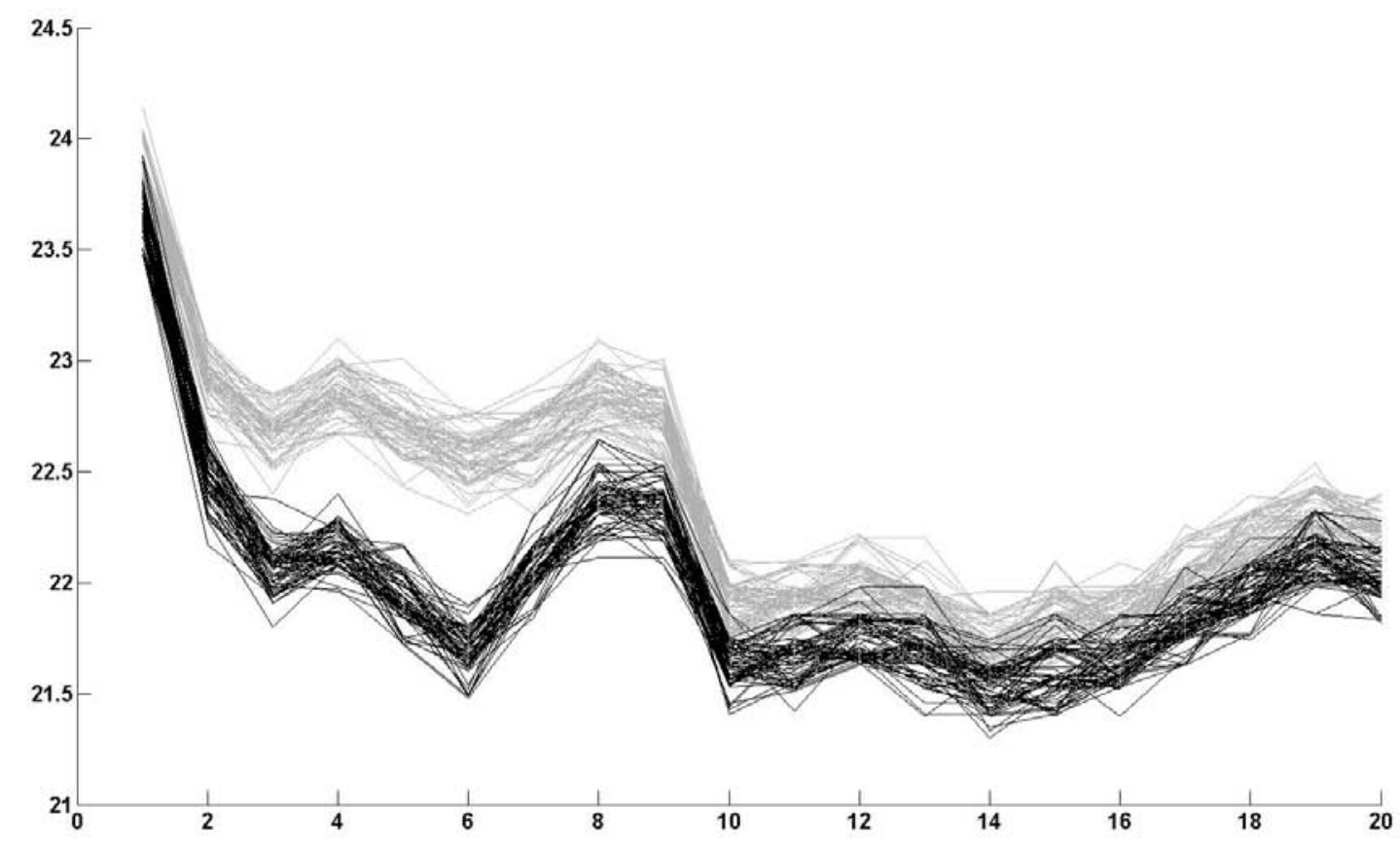

Fig. 3. Changes in temperatures for two selected regions: with contamination (grey) and without contamination (black). The vertical axis describes temperature in ${ }^{\circ} \mathrm{C}$, the horizontal axis is number of picture in the sequence.

In next step we took the same image processing procedure for each of images with the highest contrast. The choice of the most contrastive images was based on plot presented in figure 3 . We chose images number 3, 4, 5 and 6 from the sequence. Figure 4 presents algorithm of this processing. This algorithm is described above, in first processing, when mean image was taken. 


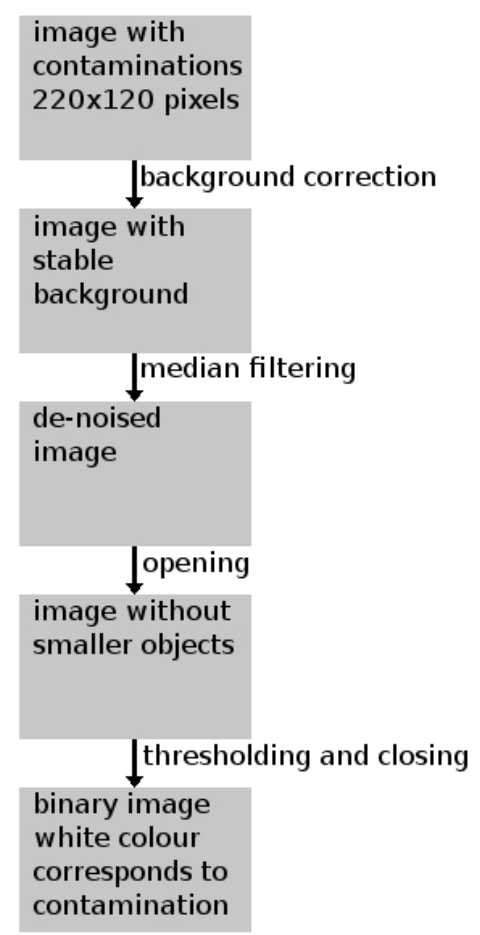

Fig. 4. Thermographic image processing algorithm

As a result we obtained four binary images in which possible contaminations are visible. We calculated product of those images to identify the most possible regions of foreign bodies. All described images are presented in figure 5 .

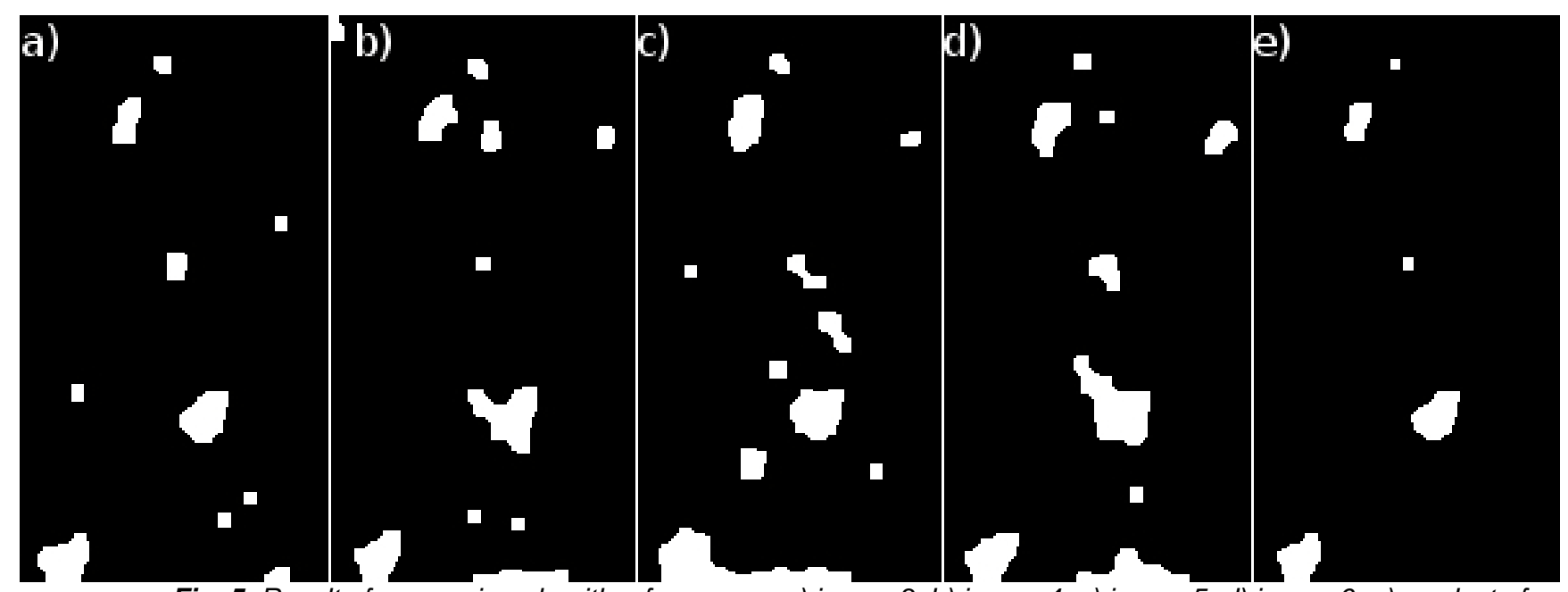

Fig. 5. Result of processing algorithm for mages: a) image 3; b) image 4; c) image 5; d) image 6; e) product of images (a), (b), (c) and (d)

In this case we get smaller number of contaminations than in first described processing. But this result is more reliable to point where the contaminations are.

This algorithm works also for sample of comestible product without contamination (left side of the images in the sequence). Then as a result we obtain binary image without any contamination. And there is no need to present this result. 


\section{Results and conclusions}

Methods of image processing allowed localizing the contaminations in comestible product. Algorithm works also for the sample of product, in which there is no contamination. Then no contaminated area is visible.

Those methods can be very useful in production processes to recognize unwanted contaminated samples. Algorithm works fast, so it can be also used in real time. Sequence of image allowed us to point areas where the probability of proper localization of contamination is very high. This makes that our results can be very useful for unwanted samples detection and raise quality of the produced product.

Methodology of thermographic images processing can be used also in many others applications, in every case where some thermal anomalies appears.

\section{Acknowledgments}

This paper was written as part of the statutory research project of the Department of Geoinformatics and Applied Computer Science, Faculty of Geology, Geophysics and Environmental Protection, AGH University of Science and Technology.

\section{REFERENCES}

[1] Bukowska-Belniak B., Leśniak A., "Application of Thermographic Research in Environmental Protection," Polish Journal of Environmental Studies, Olsztyn (Poland), Vol. 18, No. 3A, p. 38-43, 2009.

[2] Gonzalez, R.C., Woods, R.E., Digital Image Processing, Prentice Hall Inc., 2002 